\title{
Recursive Backstepping Nonlinear Control and Sliding Mode Control of a Novel Hyperchaotic Finance System
}

\author{
Pei Hu, Guoliang Cai, Lan Yao and Xiulei Fang \\ Nonlinear Scientific Research Center, Jiangsu University, Zhenjiang, Jiangsu, 212013, China \\ Email: glcai@ujs.edu.cn
}

\begin{abstract}
In this paper, the recursive backstepping nonlinear control method is proposed. Based on the Lyapunov theory, the controllers are designed to achieve the new hyperchaotic system globally, asymptotically stabilized at the equilibrium point. Furthermore, a robust control method combining backstepping and sliding mode control techniques is used to control the system to another equilibrium point. Numerical simulation results show that the proposed control schemes are effective.
\end{abstract}

Keywords-recursive backstepping control; backstepping sliding mode control; hyperchaotic finance system; Lyapunov stability theory

\section{INTRODUCTION}

Nonlinear chaotic control research is a popular problem in the nonlinear science field. Since the chaotic phenomenon in economics was first found in 1985, great impact has been imposed on the prominent economics at present. Researches on the complicated economic system by applying nonlinear methods have been fruitful [1-3]. However, with the development of economy, the old financial chaotic system can't meet the needs of the market. Therefore, more and more scholars improve it by adding an additional state variable [4, 5]. Recently, a novel financial hyperchaotic system was brought up [6] on the ground of global economic crisis arousing from 2007. The dynamical behaviors of the new system are more complex.

In this paper, we study the nonlinear control of the new hyperchaotic finance system by both recursive backstepping control and backstepping sliding mode control method. Backstepping method has become one of the important and popular approaches for nonlinear systems [7-9]. The each two backstepping method employed in this paper has its own characteristics, but both effective. Especially, the backstepping sliding mode method combines both the merits of backstepping control and sliding mode control. The Lyapunov stability theory is used to analyze the asymptotic stability at the equilibrium points. Moreover, numerical simulations are applied to verify the effectiveness of chosen controllers. By comparison, we can know that the difference of two methods and we can take corresponding way under different situations.

The rest of this article is organized as follows: In Section 2 , the new hyperchaotic finance system is introduced. In Section 3 , the recursive backstepping control is considered and numerical simulation results verify it. Then Section 4 shows backstepping sliding mode control of the hyperchaotic finance system and corresponding numerical simulation results. Finally the conclusion is given in Section 5.

\section{THE NOVEL HYPERCHAOTIC FINANCE SYSTEM}

The novel nonlinear hyperchaotic finance system [6] can be described by the following differential equation:

$$
\left\{\begin{array}{l}
\dot{x}=z+(y-a) x+w, \\
\dot{y}=1-b y-x^{2}, \\
\dot{z}=-x-c z, \\
\dot{w}=-d x y-k w,
\end{array}\right.
$$

where variable $x$ represents the interest rate, variable $y$ represents the investment demand, variable $z$ is the price exponent and variable $w$ is the average profit margin, $a, b, c$, $d, k$ are the positive parameters. When $a=0.9, b=0.2, c=$ $1.5, d=0.2$ and $k=0.17$, the system (1) shows hyperchaotic behavior. Figure 1 presents the chaotic attractor of financial hyperchaotic system.

It is easy to see that system (1) has three equilibrium points: $P_{0}(0,1 / b, 0,0), P_{1,2}\left( \pm \theta, \frac{k+a c k}{c(k-d)}, \mp \frac{\theta}{c}, \frac{d \theta(1+a c)}{c d-c k}\right)$, where ${ }_{\theta=} \sqrt{\frac{k b+a b c k}{c(d-k)}+1}$. By calculation, equilibriums $P_{0,1,2}$ are unstable saddle points in this nonlinear four-dimensional autonomous system .

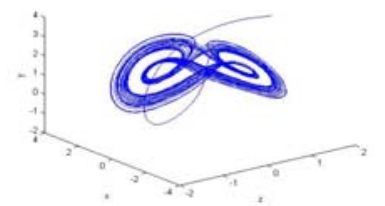

Fig. 1 The chaotic attractor of system (1)

\section{RECURSIVE BACKSTEPPING NONLINEAR CONTROL}

In this section, we will control hyperchaotic system (1) to equilibrium point $P_{0}(0,1 / b, 0,0)$ by using recursive backstepping nonlinear control method.

Consider the following nonlinear chaotic system and its responding controlled system:

$$
\begin{gathered}
\dot{x}=f(x), \\
\dot{x}=f(x)+u(t),
\end{gathered}
$$


where $x=\left(x_{1}, x_{2}, \ldots, x_{n}\right)^{\mathrm{T}}, y=\left(y_{1}, y_{2}, \ldots, y_{n}\right)^{\mathrm{T}} \in R^{n}$ are the state variables, $f: R^{n} \rightarrow R^{n}$ are differentiable function vectors, $u(t)$ is a control function and $u(0)=0$.

Let the error vector state be $e=x-x_{d}$, where $e=\left(e_{1}, e_{2}, \ldots, e_{n}\right)^{\mathrm{T}}, x_{d}=A e, A=\left(a_{i j}\right)_{n \times n}$ is a constant vector, and when $1 \leq i \leq j \leq n, a_{i j}=0$. Thus, the error dynamical system can be described as follows:

$$
\dot{e}=(A+I)^{-1}[f((A+I) e)+u(t)] .
$$

Choose the following Lyapunov function:

$$
V=\frac{1}{2} \sum_{i=1}^{n} k_{i} e_{i}^{2},
$$

then the time derivative of the Lyapunov function along the trajectory is

$$
\dot{V}=\sum_{i=1}^{n} k_{i} e_{i} \dot{e}_{i}
$$

By setting $A, u(t)$ appropriate values, $V(t)$ is positive definite and $\dot{V}(t)$ is negative definite. And then the error dynamical system (4) is asymptotically stable, namely the controlled nonlinear chaotic system (3) is asymptotically stable.

For this purpose, we have a transformation of hyperchaotic system (1) at the equilibrium point $P_{0}$ :

$$
\left\{\begin{array}{l}
X=x, \\
Y=y-1 / b \\
Z=z, \\
W=w,
\end{array}\right.
$$

then we suppose the controlled hyperchaotic finance system as follows:

$$
\left\{\begin{array}{l}
\dot{X}=Z+(Y+1 / b-a) X+W, \\
\dot{Y}=-b Y-X^{2}, \\
\dot{Z}=-X-c Z, \\
\dot{W}=-d X / b-d X Y-k W+u(t),
\end{array}\right.
$$

where $u(t)$ is external control inputs.

Let the error vector state be $e=x-x_{d}$, where $e=\left(e_{1}, e_{2}, e_{3}, e_{4}\right)^{\mathrm{T}}, x=(X, Y, Z, W), x_{d}=A e, A=\left(a_{i j}\right)_{4 \times 4}$, and when $1 \leq i \leq j \leq 4, a_{i j}=0$. Thus, the error dynamical system can be described as follows:

$$
\begin{aligned}
\dot{e}_{1} & =\left(a_{31}+1 / b-a\right) e_{1}+\left(a_{32}+a_{42}\right) e_{2}+\left(1+a_{43}\right) e_{3} \\
& +\left(1+a_{41}\right) e_{4}+a_{21} e_{1}^{2}+e_{1} e_{2}, \\
\dot{e}_{2} & =-b a_{21} e_{1}-b e_{2}-e_{1}^{2}-a_{21} \dot{e}_{1}, \\
\dot{e}_{3} & =\left(b a_{21} a_{32}-c a_{31}-1\right) e_{1}+\left(b a_{32}-c a_{32}\right) e_{2} \\
& -c e_{3}-a_{32} e_{1}^{2}+\left(a_{21} a_{32}-a_{31}\right) \dot{e}_{1},
\end{aligned}
$$

$$
\begin{aligned}
\dot{e}_{4} & =\left(b a_{21} a_{42}-b a_{21} a_{32} a_{43}+a_{43}+c a_{43}-\frac{d}{b}-k a_{41}\right) e_{1} \\
& +\left(a_{32} a_{43}-a_{42}-a_{32} a_{43}-k a_{42}\right) e_{2}+\left(c a_{43}-k a_{43}\right) e_{3} \\
& -k e_{4}+\left(a_{42}-a_{32} a_{43}-d a_{21}\right) e_{1}^{2}-d e_{1} e_{2} \\
& +\left(a_{21} a_{42}+a_{31} a_{43}-a_{41}-a_{21} a_{32} a_{43}\right) \dot{e}_{1}+u(t) .
\end{aligned}
$$

Theorem1. When setting $a_{21}=a_{42}=a_{43}=0$, $a_{41}=-b /(k d) \quad, \quad k_{1}=k_{2}=k_{3}=0 \quad, \quad k_{4}=1 \quad$, and $u(t)=-(b / k d) \dot{e}_{1}+d e_{1} e_{2}$, the controlled hyperchaotic finance system (7) is asymptotically stable at equilibrium point $(0,0,0,0)$.

Proof: Choose the following Lyapunov function

$$
V=\frac{1}{2}\left(k_{1} e_{1}^{2}+k_{2} e_{2}^{2}+k_{3} e_{3}^{2}+k_{4} e_{4}^{2}\right),
$$

which is positive definite obviously, then the time derivative of the Lyapunov function along the trajectory is

$$
\dot{V}=k_{4} e_{4}\left[-k e_{4}-d e_{1} e_{2}+(b / k d) \dot{e}_{1}+u(t)\right],
$$

when sets $a_{21}=a_{42}=a_{43}=0, a_{41}=-b /(k d), k_{1}=k_{2}=k_{3}=0$, $k_{4}=1$. Thus, the controller can be chosen as follows:

$$
u(t)=-(b / k d) \dot{e}_{1}+d e_{1} e_{2},
$$

and then we have $\dot{V}=-k e_{4}^{2}$, which is negative definite obviously. According to Lyapunov stability theory, the controlled hyperchaotic finance system (6) is asymptotically stable at equilibrium point $(0,0,0,0)$.

In the numerical simulations, the initial states of the controlled system (6) are selected as $(1,2,1,1)$, and the behaviors of the states $(X, Y, Z, W)$ of the controlled chaotic system (6) with time are displayed in Fig.2.

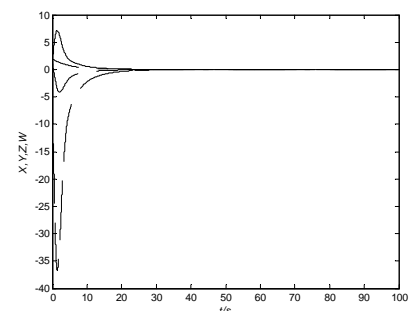

Fig.2 the time evolution of the states of the controlled system (6).

\section{BACKSTEPPING SLIDING MODE CONTROL}

In this section, we will control chaotic system (3) to equilibrium point $P_{1}\left(\theta, \frac{k+a c k}{c(k-d)},-\frac{\theta}{c}, \frac{d \theta(1+a c)}{c d-c k}\right)$ by using adaptive backstepping sliding mode control method.

At first, we have a transformation of the hyperchaotic system (1) at the equilibrium point $P_{1}$ :

$$
\left\{\begin{array}{l}
X=x-\theta, \\
Y=y-\frac{k+a c k}{c k-c d}, \\
Z=z+\frac{\theta}{c}, \\
W=w-\frac{d \theta(1+a c)}{c d-c k},
\end{array}\right.
$$


then we assume that the controlled hyperchaotic finance system is as follows:

$$
\left\{\begin{array}{l}
\dot{X}=\frac{k+a c d}{c k-c d} X+X Y+\theta Y+Z+W+u(t), \\
\dot{Y}=-2 \theta X-X^{2}-b Y+v(t), \\
\dot{Z}=-X-c Z, \\
\dot{W}=\frac{d k(1+a c)}{c d-c k} X-d X Y-d \theta Y-k W,
\end{array}\right.
$$

where $u(t), v(t)$ is external control inputs.

Definition 1. Nonlinear strict feedback systems are of the form

$$
\left\{\begin{array}{l}
\dot{\xi}_{1}=f_{1}\left(\xi_{1}\right)+g_{1}\left(\xi_{1}\right) \xi_{2}, \\
\dot{\xi}_{2}=f_{2}\left(\xi_{1}, \xi_{2}\right)+g_{2}\left(\xi_{1}, \xi_{2}\right) \xi_{3}, \\
\vdots \\
\dot{\xi}_{k-1}=f_{k-1}\left(\xi_{1}, \ldots, \xi_{k-1}\right)+g_{k-1}\left(\xi_{1}, \ldots, \xi_{k-1}\right) \xi_{k}, \\
\dot{\xi}_{k}=f_{k}\left(\xi_{1}, \ldots, \xi_{k}\right)+g_{k}\left(\xi_{1}, \ldots, \xi_{k}\right) u,
\end{array}\right.
$$

where $\xi_{1}, \ldots, \xi_{k}$ are scalars.

Obviously, the hyperchaotic system (9) is not a strict feedback system and the ordinary backstepping technique cannot be used. But after careful examination, we notice that this system is made up of two coupled subsystems as follows:

$$
\begin{aligned}
& \left\{\begin{array}{l}
\dot{X}=m X+X Y+\theta Y+Z+W+u(t), \\
\dot{Z}=-X-c Z,
\end{array}\right. \\
& \left\{\begin{array}{l}
\dot{Y}=-2 \theta X-X^{2}-b Y+v(t), \\
\dot{W}=n X-d X Y-d \theta Y-k W,
\end{array}\right.
\end{aligned}
$$

where $m=\frac{k+a c d}{c k-c d}, n=\frac{d k(1+a c)}{c d-c k}$, and each subsystem is of strict feedback form. This kind of system is called crossstrict feedback system. Since each subsystem of this hyperchaotic system is of strict feedback form, backstepping technique can be applied to each subsystem. The following design procedure is based on this idea.

Step 1. Define variable $z_{1}=Z$, and its derivative is

$$
\dot{z}_{1}=\dot{Z}=-X-c Z \text {. }
$$

Define the stabilizing function $\alpha_{1}=c_{1} z_{1}$, where $c_{1}$ is a positive constant. We choose the first Lyapunov function $V_{1}$ as

$$
V_{1}=\frac{1}{2} z_{1}^{2} \cdot
$$

Define variable $z_{2}=\dot{z}_{1}+\alpha_{1}$, then the derivative of $V_{1}$ is

$$
\dot{V}_{1}=z_{1} \dot{z}_{1}=z_{1}\left(z_{2}-\alpha_{1}\right)=z_{1} z_{2}-c_{1} z_{1}^{2} \text {. }
$$

Step 2. The derivative of $z_{2}$ is now expressed as

$$
\dot{z}_{2}=\ddot{z}_{1}+\dot{\alpha}_{1}=-\dot{X}-c \dot{Z}+c_{1} \dot{Z}=-\dot{X}+\left(c_{1}-c\right) \dot{Z} \text {. }
$$

The second Lyapunov function $V_{2}$ is chosen as follows

$$
V_{2}=V_{1}+\frac{1}{2} \sigma_{1}^{2} \text {. }
$$

where $\sigma_{1}=k_{1} z_{1}+z_{2}$ is the sliding surface with $k_{1}>0$.Then the derivative of $V_{2}$ can be derived as

$$
\begin{aligned}
\dot{V}_{2} & =\dot{V}_{1}+\sigma_{1} \dot{\sigma}_{1}=z_{1} z_{2}-c_{1} z_{1}^{2}+\sigma_{1}\left(k_{1} \dot{z}_{1}+\dot{z}_{2}\right) \\
& =z_{1} z_{2}-c_{1} z_{1}^{2}+\sigma_{1}\left[\left(k_{1}-c+c_{1}\right)(-X-c Z)\right. \\
& -(m X+X Y+\theta Y+Z+W+u(t))] .
\end{aligned}
$$

According to (16), a backstepping sliding mode control law is designed as

$$
\begin{aligned}
u(t)= & \left(k_{1}-c+c_{1}+m\right) X+\left[c\left(k_{1}-c+c_{1}\right)+1\right] Z . \\
& +X Y+\theta Y+W-h_{1}\left(\sigma_{1}+\beta_{1} \operatorname{sgn}\left(\sigma_{1}\right)\right),
\end{aligned}
$$

where $h_{1}$ and $\beta_{1}$ are positive constants. Substituting (17) into (16), the following function can be obtained

$$
\begin{aligned}
\dot{V}_{2} & =z_{1} z_{2}-c_{1} z_{1}^{2}-\sigma_{1}\left[h_{1}\left(\sigma_{1}+\beta_{1} \operatorname{sgn}\left(\sigma_{1}\right)\right)\right] . \\
& =z_{1} z_{2}-c_{1} z_{1}^{2}-h_{1} \sigma_{1}^{2}-h_{1} \beta_{1}\left|\sigma_{1}\right| .
\end{aligned}
$$

We choose a positive definite symmetric matrix $Q$ with the form

$$
Q=\left[\begin{array}{cc}
c_{1}+h_{1} k_{1}^{2} & h_{1} k_{1}-\frac{1}{2} \\
h_{1} k_{1}-\frac{1}{2} & h_{1}
\end{array}\right],
$$

and a vector $z^{\mathrm{T}}=\left[\begin{array}{ll}z_{1} & z_{2}\end{array}\right]$, then we have

$$
\begin{aligned}
z^{\mathrm{T}} Q z & =\left[\begin{array}{ll}
z_{1} & z_{2}
\end{array}\right]\left[\begin{array}{cc}
c_{1}+h_{1} k_{1}^{2} & h_{1} k_{1}-\frac{1}{2} \\
h_{1} k_{1}-\frac{1}{2} & h_{1}
\end{array}\right]\left[\begin{array}{ll}
z_{1} & z_{2}
\end{array}\right]^{\mathrm{T}} \\
& =c_{1} z_{1}^{2}+h_{1} k_{1}^{2} z_{1}^{2}+2 h_{1} k_{1} z_{1} z_{2}-z_{1} z_{2}+h_{1} z_{2}^{2} \\
& =c_{1} z_{1}^{2}-z_{1} z_{2}+h_{1} \sigma_{1}^{2} .
\end{aligned}
$$

Substituting (20) into (18), the following function can be derived

$$
\dot{V}_{2}=-z^{\mathrm{T}} Q z-h_{1} \beta_{1}\left|\sigma_{1}\right| .
$$

According to (20), we obtain

$$
|Q|=h_{1}\left(c_{1}+h_{1} k_{1}^{2}\right)-\left(h_{1} k_{1}-\frac{1}{2}\right)^{2}=h_{1}\left(c_{1}+k_{1}\right)-\frac{1}{4} .
$$

Thus, we can keep $|Q|>0$ by choosing the right values of constants $h_{1}, c_{1}$ and $k_{1}$, so $\dot{V}_{2} \leq 0$. The system is negative definite. That is $z_{1}$ and $z_{2}$ will converge to zero as $t \rightarrow \infty$. Moreover, $\lim _{t \rightarrow \infty} X=0$ and $\lim _{t \rightarrow \infty} Z=0$. Therefore the backstepping controlled subsystem is asymptotically stable .

Step 3. Define variable $z_{3}=W$, and its derivative is

$$
\dot{z}_{3}=\dot{W}=n X-d X Y-d \theta Y-k W .
$$

Define the stabilizing function $\alpha_{2}=c_{2} z_{3}$, where $c_{2}$ is a positive constant. We choose the Lyapunov function $V_{3}$ as

$$
V_{3}=\frac{1}{2} z_{3}^{2} \text {. }
$$

Define variable $z_{4}=\dot{z}_{3}+\alpha_{2}$, then the derivative of $V_{3}$ is

$$
\dot{V}_{3}=z_{3} \dot{z}_{3}=z_{3}\left(z_{4}-\alpha_{2}\right)=z_{3} z_{4}-c_{2} z_{3}^{2} \text {. }
$$

Step 4. The derivative of $z_{4}$ is now expressed as

$$
\begin{aligned}
\dot{z}_{4} & =\ddot{z}_{3}+\dot{\alpha}_{2}=n \dot{X}-d \dot{X} Y-d X \dot{Y}-d \theta \dot{Y}-k \dot{W}+c_{2} \dot{W} \\
& =n \dot{X}-d \dot{X} Y-d X \dot{Y}-d \theta \dot{Y}+\left(c_{2}-k\right) \dot{W} .
\end{aligned}
$$


The second Lyapunov function $V_{4}$ is chosen as follows

$$
V_{4}=V_{3}+\frac{1}{2} \sigma_{2}^{2} .
$$

where $\sigma_{2}=k_{2} z_{3}+z_{4}$ is the sliding surface with $k_{2}>0$.Then the derivative of $V_{4}$ can be derived as

$$
\begin{aligned}
& \dot{V}_{4}=\dot{V}_{3}+\sigma_{2} \dot{\sigma}_{2}=z_{3} z_{4}-c_{2} z_{3}^{2}+\sigma_{2}\left(k_{2} \dot{z}_{3}+\dot{z}_{4}\right) \\
& =z_{3} z_{4}-c_{2} z_{3}^{2}+\sigma_{2}[n \dot{X}-d \dot{X} Y-d(X+\theta) . \\
& \left.\left(-2 \theta X-X^{2}-b Y+v(t)\right)+\left(c_{2}+k_{2}-k\right) \dot{W}\right] .
\end{aligned}
$$

According to (28), a backstepping sliding mode control law is designed as

$$
\begin{aligned}
v(t)= & 2 \theta X+X^{2}+b Y+[d(X+\theta)]^{-1}[(n-d Y) \dot{X} \\
& \left.+\left(c_{2}+k_{2}-k\right) \dot{W}-h_{2}\left(\sigma_{2}+\beta_{2} \operatorname{sgn}\left(\sigma_{2}\right)\right)\right],
\end{aligned}
$$

where $h_{2}$ and $\beta_{2}$ are positive constants. Substituting (17) into (16), the following function can be obtained

$$
\begin{aligned}
\dot{V}_{4} & =z_{3} z_{4}-c_{2} z_{3}^{2}-\sigma_{2}\left[h_{2}\left(\sigma_{2}+\beta_{2} \operatorname{sgn}\left(\sigma_{2}\right)\right)\right] \\
& =z_{3} z_{4}-c_{2} z_{3}^{2}-h_{2} \sigma_{2}^{2}-h_{2} \beta_{2}\left|\sigma_{2}\right| .
\end{aligned}
$$

We choose a positive definite symmetric matrix $P$ with the form

$$
P=\left[\begin{array}{cc}
c_{2}+h_{2} k_{2}^{2} & h_{2} k_{2}-\frac{1}{2} \\
h_{2} k_{2}-\frac{1}{2} & h_{2}
\end{array}\right],
$$

and a vector $z^{\prime \mathrm{T}}=\left[\begin{array}{ll}z_{3} & z_{4}\end{array}\right]$, then we have

$$
\begin{aligned}
z^{\prime \mathrm{T}} P z^{\prime} & =c_{2} z_{3}^{2}+h_{3} k_{2}^{2} z_{3}^{2}+2 h_{2} k_{2} z_{3} z_{4}-z_{3} z_{4}+h_{2} z_{4}^{2} \\
& =c_{2} z_{3}^{2}-z_{3} z_{4}+h_{2} \sigma_{2}^{2} .
\end{aligned}
$$

Substituting (32) into (30), the following function can be derived

$$
\dot{V}_{4}=-z^{\mathrm{T}} P z^{\prime}-h_{2} \beta_{2}\left|\sigma_{2}\right| \text {. }
$$

According to (32), we obtain

$$
|P|=h_{2}\left(c_{2}+h_{2} k_{2}^{2}\right)-\left(h_{2} k_{2}-\frac{1}{2}\right)^{2}=h_{2}\left(c_{2}+k_{2}\right)-\frac{1}{4} .
$$

Thus, we can keep $|P|>0$ by choosing the right values of constants $h_{2}, c_{2}$ and $k_{2}$, so $\dot{V}_{4} \leq 0$. The system is negative definite. That is $z_{3}$ and $z_{4}$ will converge to zero as $t \rightarrow \infty$. Moreover, $\lim _{t \rightarrow \infty} Y=0$ and $\lim _{t \rightarrow \infty} W=0$. Therefore the backstepping controlled subsystem is asymptotically stable .

As a result, the stability of the proposed backstepping sliding mode control system (10) can be guaranteed.

In the numerical simulations, the initial states of the controlled system (10) are selected as $(1,2,1,1)$, and $k_{1}=h_{1}=c_{1}=2, k_{2}=h_{2}=c_{2}=3, \beta_{1}=\beta_{2}=1$. Figure 3 present us the behaviors of the states $(X, Y, Z, W)$ of the controlled chaotic system (10) with time.

\section{CONCLUSION}

In this paper, the recursive backstepping nonlinear control and backstepping sliding mode control finance system proposed nearly are studied. The hyperchaotic system is controlled to its equilibrium points by designing appropriate controllers with two methods respectively. Numerical simulations demonstrate the efficiency of both the proposed control schemes. Then, we can see where the worth of the study here: it can provide reference for government regulation when the economic crisis happens.

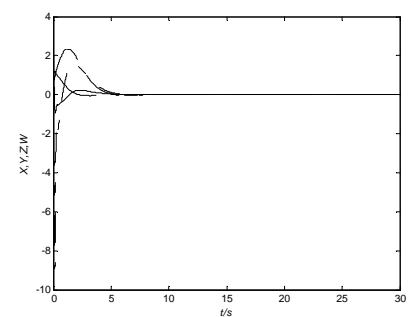

Fig.3 the time evolution of the states of the controlled system (11)

\section{ACKNOWLEDGMENT}

This work was supported by the National Nature Science foundation of China (Grants 51276081, 71073072), the Society Science Foundation from Ministry of Education of China (Grants 12YJAZH002, 08JA790057), the Project Funded by The Priority Academic Program Development of Jiangsu Higher Education Institutions, the Advanced Talents' Foundation of Jiangsu University (Grant 07JDG054), and the Students' Research Foundation of Jiangsu University (Grants 11A179, Y11A079).

\section{EFERENCE}

[1] B.C. Trevor, A. Katie, M. Peter, "Using argumentation to model agent decision making in economic experiments," Autonomous Agents and Multi-agent Systems, vol. 25, pp.154-167, 2012.

[2] G.L. Cai, H.J. Yu, Y.X. Li, "Localization of compact invariant sets of a modified nonlinear finance chaotic system," Nonlinear Dyn., vol.69, pp. 2269-2275, 2012.

[3] M.Z. Yang, G.L. Cai, "Chaos Control of a Non-linear Finance System,” J. Uncertain Syst., vol. 5, pp. 263-270, 2011.

[4] D. Sara, M. H. Reza, "Control of a fractional-order economical system via sliding mode," Physica A, vol. 389, pp. 2434-2442, 2010.

[5] G.L. Cai, M.Z. Yang, "Globally exponentially attractive set and synchronization of a novel three-dimensional chaotic finance system," Proc. Third Inter. Conf. Inform. Comp. Sci. 2010, 2, pp.7073.

[6] H.J. Yu, G.L. Cai, Y.X. Li, "Dynamic analysis and control of a new hyperchaotic finance system," Nonlinear Dyn., vol.67, pp. 2171-2182, 2012.

[7] G.L. Cai, P. Hu, Y.X. Li, "Modified function lag projective synchronization of a financial hyperchaotic system," Nonlinear Dyn., vol.69, pp. 1457-1464, 2012.

[8] J.H. Park, "Synchronization of Genesio chaotic system via backstepping approach," Chaos Solitons Fractal, vol. 27, pp.13691375, 2006.

[9] G.H. Li, S.P. Zhou, K. Yang, "Generalized projective synchronization between two different chaotic systems using active backstepping control," Phys. Lett. A, vol. 355, pp. 326-330, 2006. 